\title{
ORIGINAL RESEARCH \\ Using Electronic Health Records to Examine Disease Risk in Small Populations: Obesity Among American Indian Children, Wisconsin, 2007-2012
}

\author{
Emily J. Tomayko, PhD, RD; Bethany A. Weinert, MD; Liz Godfrey, MS; \\ Alexandra K. Adams, MD, PhD; Lawrence P. Hanrahan, PhD, MS
}

\begin{abstract}
Suggested citation for this article: Tomayko EJ, Weinert BA, Godfrey L, Adams AK, Hanrahan LP. Using Electronic Health Records to Examine Disease Risk in Small Populations: Obesity Among American Indian Children, Wisconsin, 2007-2012. Prev Chronic Dis 2016;13:150479. DOI: http://dx.doi.org/10.5888/ pcd13.150479.
\end{abstract}

\section{PEER REVIEWED}

\section{Abstract}

\section{Introduction}

Tribe-based or reservation-based data consistently show disproportionately high obesity rates among American Indian children, but little is known about the approximately $75 \%$ of American Indian children living off-reservation. We examined obesity among American Indian children seeking care off-reservation by using a database of de-identified electronic health records linked to community-level census variables.

\section{Methods}

Data from electronic health records from American Indian children and a reference sample of non-Hispanic white children collected from 2007 through 2012 were abstracted to determine obesity prevalence. Related community-level and individual-level risk factors (eg, economic hardship, demographics) were examined using logistic regression.

\section{Results}

The obesity rate for American Indian children $(n=1,482)$ was double the rate among non-Hispanic white children $(\mathrm{n}=81,042)$ $(20.0 \%$ vs $10.6 \%, P<.001)$. American Indian children were less likely to have had a well-child visit $(55.9 \%$ vs $67.1 \%, P<.001)$ during which body mass index (BMI) was measured, which may partially explain why BMI was more likely to be missing from American Indian records $(18.3 \%$ vs $14.6 \%, P<.001)$. Logistic regression demonstrated significantly increased obesity risk among American Indian children (odds ratio, 1.8; 95\% confidence interval, 1.6-2.1) independent of age, sex, economic hardship, insurance status, and geographic designation.

\section{Conclusion}

An electronic health record data set demonstrated high obesity rates for nonreservation-based American Indian children, rates that had not been previously assessed. This low-cost method may be used for assessing health risk for other understudied populations and to plan and evaluate targeted interventions.

\section{Introduction}

Small population subgroups are often excluded from large-scale studies or surveys, making it difficult to assess health risk in these groups. Electronic health record (EHR) data sets may be wellsuited to address these data gaps; a 2013 report to the US Department of Health and Human Services highlighted the feasibility of this approach and called for examination of underserved populations using this methodology (1). One such group is American Indian children who live outside of reservations or other tribal lands. Although data are available for American Indian children living on reservation through the Indian Health Service (IHS), less is known about children who live and seek health care outside of IHS. Data characterizing the differences in aspects of health and health care in this group and other subpopulations can lead to improvements in health care quality, evidence-based research, and public health approaches.

IHS is responsible for the health care of American Indian members of federally recognized tribes and serves approximately 2.2 
million patients (2). However, approximately 2.9 million people self-identify as either American Indian or Alaska Native alone, and an additional 2.3 million self-identify as American Indian or Alaska Native in combination with other races, suggesting a significant proportion of this population may not receive medical care through IHS. Moreover, 78\% of the American Indian and Alaska Native alone-or-in-combination population live outside of American Indian/Alaska Native reservations (3). Although nonreservation urban IHS clinics exist, most care that American Indians living outside of reservations receive is from the private sector (4).

Although little has been reported about American Indian children seeking care outside of reservations, the obesity prevalence among American Indian children living on reservations is known to be among the highest of all racial/ethnic groups (5-7) and may be related to socioeconomic and environmental factors. For example, the National Longitudinal Study of Adolescent to Adult Health (Add Health) included a subsample of American Indian teenagers that showed high rates of obesity associated with poverty and stress (8). However, the obesity prevalence or specific determinants of obesity in children seeking health care outside of reservation-based or IHS services are less well characterized, particularly among younger children.

The purpose of this study was to use the Public Health Information Exchange (PHINEX), a data set that links EHR data to community-level variables, to describe childhood obesity among American Indian children seeking health care in Wisconsin. The primary aim was to address a gap regarding the prevalence and risk profile of American Indian children seeking care outside of reservation areas. The secondary aim was to examine the usefulness of EHRs to examine disease risk in a small population as an alternative to resource-intensive surveys and data collection methods. We hypothesized this EHR-based approach would identify high rates of obesity among nonreservation-based American Indian children and demonstrate the usefulness of EHR data as a costeffective and efficient resource for risk estimates among small populations.

\section{Methods}

The University of Wisconsin (UW) PHINEX database links deidentified EHR data to community-level variables (eg, neighborhood socioeconomic and demographic variables), as described previously (9). PHINEX includes records of patient encounters that occurred from 2007 through 2012 at UW Health primary care clinics serving mostly south-central Wisconsin. Block group level data (ie, community-level data, typically containing 600-3,000 people) were extracted from 5-year estimates of the US Census American Community Survey 2007-2012 and from Esri Business
Analyst (Esri). The study was reviewed and approved by the UW-Madison School of Medicine and Public Health institutional review board under Protocol M2009-1273, titled "Family Medicine/Public Health Data Exchange."

Patient records were selected from the PHINEX database for patients who had at least one encounter from January 1, 2007, through December 31,2012, while they were between the ages of 2 and 17 years and who were identified as American Indian. We included all patients who identified as American Indian for race and who indicated either Hispanic or non-Hispanic ethnicity. Patients in the same age range who were identified as non-Hispanic white were included as the reference group. In the PHINEX database, $98.7 \%$ of patients selected only one response for race (with ethnicity reported as a separate category). Race information was listed as "unknown" for $5.2 \%$ of patients, and missing race information was less than $1 \%$. Although UW Health clinics may differ in the way race/ethnicity data are collected (eg, collected at registration desk vs in-office by nurse), the standard practice is for the patient (or parent or guardian) to self-identify race/ethnicity on the clinic intake form. We excluded patient records that did not meet age or race/ethnicity criteria and performed complete case analysis for body mass index (BMI) $\left(\mathrm{kg} / \mathrm{m}^{2}\right)$ and other individuallevel variables.

Patient data were collected by clinic staff during documented primary care encounters (family medicine and pediatrics). Variables included in this study were age, BMI, sex, self-reported or parent-reported race/ethnicity, number and type of encounters, and insurance type. Number of encounters was calculated as visits per patient year (number of visits divided by number of years for which patients had at least one visit during the study period). BMI measures for children were plotted on age-specific and sex-specific growth charts to determine BMI percentile according to the CDC 2000 charts $(<5$ th percentile $=$ underweight, 5 th to $<85$ th percentile $=$ normal weight, 85 th to $<95$ th percentile $=$ overweight, $\geq 95$ th percentile $=$ obese) (10). If multiple patient encounters were available, the most recently recorded BMI was used.

Before de-identification, patients were linked to census block groups by using their geocoded address of residence. Two community-level variables were used: geographic designation (ie, urban, rural, suburban) and economic hardship index (EHI) score by census block group as a measure of community-level socioeconomic status (11). The geographic designation was based on the acquisition of census block groups using geocoded patient addresses. Esri's Tapestry demographic segmentation methodology was then applied, which divides US residential areas into distinctive segments on the basis of socioeconomic and demographic characteristics to provide an accurate description of neighbor-

The opinions expressed by authors contributing to this journal do not necessarily reflect the opinions of the U.S. Department of Health and Human Services, the Public Health Service, the Centers for Disease Control and Prevention, or the authors' affiliated institutions. 
hoods. The calculation of EHI has been described previously in Appendix 1 of Nathan and Adams (12) and was normalized for all Wisconsin block groups for this analysis. The economic hardship index was calculated at the census block group level and consists of 6 measures: crowded housing (percentage of housing units with more than 1 person per room), poverty (percentage of households below the federal poverty level), unemployment (percentage of people aged 16 years or older who are unemployed), education (percentage of people aged 25 years or older without a high school education), dependency (percentage of population younger than 18 years or older than 64 years), and per capita income. Scores can range from 0 to 100 , with 100 indicating the highest hardship (ie, crowded housing, poverty, unemployment, large number of dependents, low per capita income, and low education level) (12).

Statistical analysis was performed using SAS software, version 9.3 (SAS Institute, Inc). Variables were compared between the American Indian population and the non-Hispanic white population (reference population) within the PHINEX database using Pearson $\chi^{2}$ statistical testing or the Wilcoxon test to examine group differences. Odds ratios (ORs) were calculated using stepwise logistic regression adjusted for EHI, insurance status, ethnicity, sex, age, and geographic designation. A drop-in-deviance test was used to test the effect of race: the full model included an effect for American Indian as well as interaction terms between American Indian and covariates, and the reduced model included only covariates. The Akaike information criterion was used for model selection.

\section{Results}

For all available records, no data were missing for age, sex, or insurance status. BMI measurement in the electronic record was more likely to be missing for American Indian patients than for non-Hispanic white patients $(18.3 \%$ vs $14.6 \%, P<.001)$. There were no differences between American Indian and non-Hispanic white patients in missing data related to EHI $(9.3 \%$ vs $8.6 \%, P=$ .32 ) or geographic designation $(9.3 \%$ vs $8.5 \%, P=.32)$.

The study included 1,482 American Indian patients and 81,042 non-Hispanic white patients with documented primary care visits between 2007 and 2012. Of the American Indian patients, 66\% were Hispanic. American Indian children were $1.4 \%$ of the total population within the PHINEX database. All demographic variables differed significantly $(P<.001)$ between American Indian and non-Hispanic white populations except for sex (Table 1). A higher percentage of children aged 5 to 11 years were American Indian (51.4\% vs 41.9\%). More non-Hispanic white children had commercial insurance $(83.7 \%)$ than American Indian children (53.7\%). More American Indian children than non-Hispanic white children had Medicaid (42.1\% vs $14.7 \%$ ) or no health insurance
(4.1\% vs 1.6\%). More American Indian children had an EHI classification greater than $25(52.8 \%$ vs $40.6 \%)$ than non-Hispanic white children, indicating a higher degree of economic hardship.

American Indian children had slightly fewer total clinic visits per person-year than non-Hispanic white children (mean [SD], 2.5 [1.9] vs mean [SD], 2.6 [1.9]; $P<.01$ ) (Table 2). Moreover, despite the similar number of visits per person-year, American Indian children had significantly fewer well-child visits $(55.9 \%$ vs $67.1 \%$, $P<.001)$. The clinic visits for American Indian and non-Hispanic white children appeared to be of similar medical complexity; American Indian children had a comparable number of diagnoses per visit compared with non-Hispanic white children (mean [SD], 1.9 [0.7] for both; $P=.20$ ).

American Indian children had nearly double the rate of obesity compared with the non-Hispanic white population $(20.0 \%$ vs $10.6 \%, P<.001)$ and a higher rate of overweight $(14.8 \% \mathrm{vs}$ $12.7 \%, P<.001$ ) (Table 1). A drop-in-deviance test indicated a significant effect of American Indian race on obesity $(P<.001)$. The Akaike information criterion indicated that a model with only a main effect for American Indian race provided a better fit than a model with American Indian race as well as interaction terms between American Indian race and the covariates. In a model that included race/ethnicity, age, sex, economic hardship, insurance status, and geographic designation, logistic regression analysis for obesity showed the odds of obesity were significantly higher for children self-identified as American Indian, those for whom Medicaid or Medicare was listed for health insurance, children living in a census block group with an EHI of 25 or greater, children aged 12 to 17 , and male children (Table 3 ).

\section{Discussion}

Our study demonstrates the usefulness of an electronic health record database to analyze disease risk in a small population. By using this method, we addressed a gap in the literature by examining childhood obesity in American Indian children receiving care outside of IHS or tribal clinics, a population typically excluded from national and local health surveys.

By using the PHINEX database, our analysis demonstrated a higher rate of overweight and obesity in American Indian children compared with a reference sample of non-Hispanic white children seen at the same clinics. The obesity disparity was markedly greater than the disparity in overweight between non-Hispanic white and American Indian children. These patterns are similar to those identified in a data set of reservation-based American Indian children in Wisconsin (aged 3-8 y) of 20.0\% overweight and 25.2\% obese (13). In addition, a comprehensive review of American Indi-

The opinions expressed by authors contributing to this journal do not necessarily reflect the opinions of the U.S. Department of Health and Human Services, the Public Health Service, the Centers for Disease Control and Prevention, or the authors' affiliated institutions. 
an children in tribal communities nationwide identified high rates of both overweight and obesity, although the rates were highly variable among populations (14). As in other minority communities, the reasons for the increased prevalence among American Indians are complex and include poverty, racism, historic trauma, rural isolation, urban loss of community, stress, lack of access to healthy foods and physical activity opportunities, and safety issues that may prevent physical activity $(2,15-18)$. In addition, American Indian children are at increased risk for obesity-related chronic diseases such as diabetes and cardiovascular diseases, which are observed at high rates in this population $(19,20)$.

In our study, American Indian children were also more likely to have Medicaid, to live in urban settings, and to have higher economic hardship than non-Hispanic white children. In our regression analysis, self-report as American Indian, EHI classification of 25 or greater, and use of Medicaid or Medicare insurance significantly increased the odds of obesity. Other previously reported risk factors also were found to be significant in our analysis, including increased odds of obesity for older (12-17 y) and male children (21).

Our previous work has demonstrated the PHINEX database to be both representative of the state of Wisconsin (9) and able to determine obesity rates with precision, particularly in comparison to large national data sets, such as the National Health and Nutrition Examination Survey (NHANES) (22). Although comparison rates are available for non-Hispanic white children in NHANES, American Indian children are typically included only in the "All Race" category in NHANES reports from 1999 through 2012 $(23,24)$. Data from the 2011 Pediatric Nutrition Surveillance Survey (PedNSS) reported national rates of overweight of $20.1 \%$ and obesity rates of $20.8 \%$ for American Indian/Alaska Native children older than 2 years of age (6). However, these data were collected primarily from the Special Supplemental Nutrition Program for Women, Infants, and Children (WIC) records $(87.0 \%$ of records), which included only low-income children aged 5 years or younger and therefore excluded older children and children from higher-income families. Another difficulty with using the PedNSS as a reference population is that American Indian data were provided by 7 reservation-based tribal organizations nationwide, and nonreservation-based American Indian children were not sampled.

IHS collects data on childhood overweight and obesity only for patients who use IHS clinics (ie, reservation or nearreservation-based care), whereas many other studies that included American Indian data were conducted in small geographic areas or represent nonsurveillance studies (14). Add Health focused on teenagers and found that $76.8 \%$ of participants in their subsample were overweight or obese. The Add Health study required blood sample collection and direct measurement of BMI, which can be costly and time-consuming outside of a health care setting (8). Therefore, EHR-based approaches like the one described in our study represent an effective approach to monitor childhood obesity rates in nonreservation-based American Indian children and other small populations.

A previous report identified few data sets for American Indian populations with sample sizes over 200 and cited, among multiple barriers, geographic distribution of people living in nonreservation-based urban or rural areas and failure to collect race/ethnicity information for several large data sets (25). Rather than increasing the sample size in large federal studies or other resource-intensive approaches, using EHRs to analyze a small population has several advantages. In addition to being a rapid and inexpensive method of data collection, EHRs also provide the capacity for data set linkage and the ability to access typically excluded populations, such as the nonreservation-based American Indian children described here. For example, future research could include a de-identified database linking child and maternal health records within the PHINEX database, because maternal BMI is known to be a predictor of a child's weight. In addition, this database could be used for other groups, such as Asian minority populations and refugee groups. EHRs also offer opportunities for quality improvement in health care, such as identifying the need for consistent obesity screening protocols. Moreover, EHR data sets such as PHINEX provide a larger sample than could be easily collected otherwise. EHR data sets ultimately allow for greater surveillance of population health and risk factors at the local level (eg, census block group), which provides an unprecedented opportunity for communities to detect local health hot spots and address them with local community-level interventions. IHS data typically are anonymous to protect tribal identity, thereby preventing the examination of this local variation. Comparison of the children included in this study who sought care outside of IHS with children seeking care at IHS or tribal clinics would allow for a more complete examination of obesity determinants and represents a collaboration we hope to pursue in the future.

Our study has several limitations. The overall sample size of American Indian children was considerably smaller than that of non-Hispanic white children; however, the events of interest, overweight and obesity, were not rare events, and it is therefore unlikely that our rates were biased (26). Our analyses relied on selfreported racial and ethnic classification, and protocols for collecting this information vary among the clinics; previous reports have suggested that misclassification of American Indian patients as another race or ethnicity is a significant problem (27-29). Despite these facts, our analysis included approximately 1,500 American

The opinions expressed by authors contributing to this journal do not necessarily reflect the opinions of the U.S. Department of Health and Human Services, the Public Health Service, the Centers for Disease Control and Prevention, or the authors' affiliated institutions. 
Indian children and is the largest analysis examining childhood obesity in this particular population to date. Patients with missing data were dropped from the analysis, and this could potentially bias results. In addition, BMI was more likely to be missing for American Indian children than for non-Hispanic white children in the PHINEX database, which may be because American Indian children had significantly fewer well-child visits during which BMI is usually calculated. This information could be used to educate clinicians to improve the collection and reporting of these data and to reinforce the importance of supporting American Indian families in seeking preventive health care (eg, well-child visits). Although several factors probably contribute to the significantly higher rates of obesity for American Indian children than for non-Hispanic white children, including socioeconomic status and environmental factors, no causative factors could be determined from this study. Finally, there may have been selection bias toward children who are more likely to attend clinic visits, such as children who have family members with sufficient schedule flexibility and reliable transportation. Despite these limitations, increased obesity prevalence was found in this American Indian population and at a similar magnitude found in previous studies.

More research is needed to address important concepts of race and place in determining risk for childhood obesity and other health outcomes. It remains unclear whether American Indian children seeking care outside of tribal or IHS clinics more closely resemble children in the geographic area in which they live (such as through shared experience of economic hardship measured at the community or census block level) or align more closely with their reservation-based counterparts. This study also emphasizes the importance of standardizing the collection of race and ethnicity information in electronic medical records to decrease misclassification and missing information for future analysis.

EHR-based analysis can inform clinicians and other health care providers regarding disparities, not only in health outcomes but also in the provision of health care (eg, attendance at well-child visits) for underserved populations while directing future intervention research to address these issues. By using EHRs, we demonstrated that American Indian children seeking care outside of IHS or tribal clinics in Wisconsin experience overweight and obesity at significantly higher rates than non-Hispanic white children. Use of EHRs is a cost-effective way to examine health risks in other understudied populations.

\section{Acknowledgments}

This project was supported by the Clinical and Translational Science Award program, through the National Institutes of Health (NIH) National Center for Advancing Translational Sciences
(UL1TR000427). E.J.T. and B.A.W. were supported through NIH T32 training grants to the University of Wisconsin (UW) Department of Nutritional Sciences (5T32DK007665) and the UW Department of Family Medicine and Community Health (T32HP10010), respectively. The content is solely the responsibility of the authors and does not necessarily represent the official views of the NIH. We thank Dr John Kloke for providing statistical support for this study.

\section{Author Information}

Corresponding Author: Lawrence P. Hanrahan, PhD, MS, University of Wisconsin, School of Medicine and Public Health, Department of Family Medicine and Community Health, 1100 Delaplaine Ct, Madison, WI 53715. Telephone: 608-263-5846. Email: larry.hanrahan@fammed.wisc.edu.

Author Affiliations: Emily J. Tomayko, University of Wisconsin, College of Agricultural and Life Sciences, Department of Nutritional Sciences, Madison, Wisconsin; Bethany A. Weinert, University of Wisconsin, School of Medicine and Public Health, Department of Pediatrics and Department of Family Medicine and Community Health, Madison, Wisconsin; Liz Godfrey, UW Health, Madison, Wisconsin; Alexandra K. Adams, University of Wisconsin, School of Medicine and Public Health, Department of Family Medicine and Community Health, Madison, Wisconsin.

\section{References}

1. Devers K, Gray B, Ramos C, Shah A, Blavin F, Waidmann T. The feasibility of using electronic health data for research on small populations. Washington (DC): Urban Institute; 2013.

2. Castor ML, Smyser MS, Taualii MM, Park AN, Lawson SA, Forquera RA. A nationwide population-based study identifying health disparities between American Indians/Alaska Natives and the general populations living in select urban counties. Am J Public Health 2006;96(8):1478-84.

3. Norris T, Vines PL, Hoeffel EM. The American Indian and Alaska Native population: 2010. 2010 Census Briefs. Washington (DC): US Census Bureau; 2012.

4. Rhoades ER. American Indians and the private health care sector: the growing use of private care by Indians has implications for patients, providers, and policymakers. West J Med 2002;176(1):7-9.

5. Dalenius KBE, Smith B, Polhamus B, Grummer-Strawn L. Pediatric nutrition surveillance 2010 report. Atlanta (GA): US Department of Health and Human Services, Centers for Disease Control and Prevention; 2012.

The opinions expressed by authors contributing to this journal do not necessarily reflect the opinions of the U.S. Department of Health and Human Services, the Public Health Service, the Centers for Disease Control and Prevention, or the authors' affiliated institutions. 
6. Pan L, May AL, Wethington H, Dalenius K, Grummer-Strawn LM. Incidence of obesity among young US children living in low-income families, 2008-2011. Pediatrics 2013; 132(6):1006-13.

7. Hearst MO, Biskeborn K, Christensen M, Cushing C. Trends of overweight and obesity among white and American Indian school children in South Dakota, 1998-2010. Obesity (Silver Spring) 2013;21(1):E26-32.

8. Marley TL, Metzger MW. A longitudinal study of structural risk factors for obesity and diabetes among American Indian young adults, 1994-2008. Prev Chronic Dis 2015;12:E69.

9. Guilbert TW, Arndt B, Temte J, Adams A, Buckingham W, Tandias A, et al. The theory and application of UW ehealthPHINEX, a clinical electronic health record-public health information exchange. WMJ 2012;111(3):124-33.

10. Kuczmarski RJ, Ogden CL, Grummer LM, Flegal KM, Guo SS, Wei R, et al. CDC growth charts: United States, advance data from vital and health statistics. Advance Data 2000; 314:2-27.

11. Montiel L, Nathan R, Wright D. An update on urban hardship. Albany (NY): The Nelson A. Rockefeller Institute of Government; 2004.

12. Nathan R, Adams C. Four perspectives on urban hardship. Polit Sci Q 1989;104(3):483-508.

13. Adams A, Prince R. Correlates of physical activity in young American Indian children: lessons learned from the Wisconsin Nutrition and Growth Study. J Public Health Manag Pract 2010;16(5):394-400.

14. Schell LM, Gallo MV. Overweight and obesity among North American Indian infants, children, and youth. Am J Hum Biol 2012;24(3):302-13.

15. Walters KL, Simoni JM. Reconceptualizing native women's health: an "indigenist" stress-coping model. Am J Public Health 2002;92(4):520-4.

16. Braveman P, Egerter S, An J, Williams D. Race and socioeconomic factors affect opportunities for better health. Washington (DC): Robert Wood Johnson Foundation; 2009. http:// http://www.commissiononhealth.org/PDF/506edea1f $160-4728-9539-$ a b a 2357047 e $3 /$ Is s u e \% 20 Brief $\% 205 \% 20$ A pri $1 \% 2009 \% 20$ $\% 20$ Race $\% 20$ and $\% 20$ Socioeconomic $\% 20$ Factors.pdf. Accessed January 13, 2016.

17. Braveman P, Egerter S, Barclay C. What shapes health-related behaviors? The role of social factors. Washington (DC): Robert Wood Johnson Foundation; 2011. http://www.rwjf.org/ content/dam/farm/reports/issue_briefs/2011/rwjf70442. Accessed January 13, 2016.
18. Lillie-Blanton M, Roubideaux Y. Understanding and addressing the health care needs of American Indians and Alaska Natives. Am J Public Health 2005;95(5):759-61.

19. Burrows NR, Geiss LS, Engelgau MM, Acton KJ. Prevalence of diabetes among Native Americans and Alaska Natives, 1990-1997: an increasing burden. Diabetes Care 2000; 23(12):1786-90.

20. Galloway JM. Cardiovascular health among American Indians and Alaska Natives: successes, challenges, and potentials. Am J Prev Med 2005;29(5,Suppl 1):11-7.

21. Ng M, Fleming T, Robinson M, Thomson B, Graetz N, Margono C, et al. Global, regional, and national prevalence of overweight and obesity in children and adults during 1980-2013: a systematic analysis for the Global Burden of Disease Study 2013. Lancet 2014;384(9945):766-81.

22. Flood TL, Zhao YQ, Tomayko EJ, Tandias A, Carrel AL, Hanrahan LP. Electronic health records and community health surveillance of childhood obesity. Am J Prev Med 2015; 48(2):234-40.

23. Ogden CL, Carroll MD, Kit BK, Flegal KM. Prevalence of obesity and trends in body mass index among US children and adolescents, 1999-2010. JAMA 2012;307(5):483-90.

24. Ogden CL, Carroll MD, Kit BK, Flegal KM. Prevalence of childhood and adult obesity in the United States, 2011-2012. JAMA 2014;311(8):806-14.

25. Gaps and strategies for improving AI/AN/NA Data. Final report prepared for the US Department of Health and Human Services, Office of the Assistant Secretary for Planning and Evaluation, Office of Human Services Policy; 2007. https:// aspe.hhs.gov/sites/default/files/pdf/74926/report.pdf. Accessed January 5, 2016.

26. King G, Zeng L. Logistic regression in rare events data. Polit Anal 2001;9(2):137-63.

27. Rhoades DA. Racial misclassification and disparities in cardiovascular disease among American Indians and Alaska Natives. Circulation 2005;111(10):1250-6.

28. Harwell TS, Hansen D, Moore KR, Jeanotte D, Gohdes D, Helgerson SD. Accuracy of race coding on American Indian death certificates, Montana 1996-1998. Public Health Rep 2002;117(1):44-9.

29. Bertolli J, Lee LM, Sullivan PS;AI/AN Race /Ethnicity Data Validation Workgroup. Racial misidentification of American Indians/Alaska Natives in the HIV/AIDS Reporting Systems of five states and one urban health jurisdiction, U.S., 1984-2002. Public Health Rep 2007;122(3):382-92.

The opinions expressed by authors contributing to this journal do not necessarily reflect the opinions of the U.S. Department of Health and Human Services, the Public Health Service, the Centers for Disease Control and Prevention, or the authors' affiliated institutions. 


\section{Tables}

Table 1. Demographic Characteristics, American Indian and Non-Hispanic White Children, ${ }^{a}$ University of Wisconsin Clinics, 2007-2012

\begin{tabular}{|c|c|c|}
\hline Characteristic & American Indian, n (\%) & Non-Hispanic White, n (\%) \\
\hline Total & $1,482(1.4)$ & $81,042(79.3)^{b}$ \\
\hline \multicolumn{3}{|l|}{ Age, y } \\
\hline $2-4$ & $266(18.0)$ & $16,784(20.7)$ \\
\hline $5-11$ & $762(51.4)$ & 33,945 (41.9) \\
\hline $12-17$ & $454(30.6)$ & $30,313(37.4)$ \\
\hline Male & $776(52.4)$ & $42,325(52.2)$ \\
\hline \multicolumn{3}{|c|}{ Body mass index ${ }^{c}$} \\
\hline Underweight & $28(1.9)$ & 2,399 (3.0) \\
\hline Normal weight & $668(45.1)$ & $47,930(59.1)$ \\
\hline Overweight & $219(14.8)$ & $10,289(12.7)$ \\
\hline Obese & $296(20.0)$ & $8,576(10.6)$ \\
\hline Data missing & $271(18.3)$ & $11,848(14.6)$ \\
\hline \multicolumn{3}{|l|}{ Health insurance } \\
\hline Commercial & $796(53.7)$ & $67,839(83.7)$ \\
\hline Medicaid & $624(42.1)$ & $11,879(14.7)$ \\
\hline Medicare & $1(0.1)$ & $12(0.01)$ \\
\hline No insurance & $61(4.1)$ & $1,307(1.6)$ \\
\hline \multicolumn{3}{|c|}{ Economic hardship index ${ }^{d}$} \\
\hline$<20$ & $143(9.6)$ & $8,190(10.1)$ \\
\hline $20-<25$ & $419(28.3)$ & $33,063(40.8)$ \\
\hline$\geq 25$ & $782(52.8)$ & $32,862(40.6)$ \\
\hline Data missing & $138(9.3)$ & $6,927(8.6)$ \\
\hline \multicolumn{3}{|c|}{ Geographic designation } \\
\hline Urban & $655(44.2)$ & $15,211(18.8)$ \\
\hline Suburban & $538(36.3)$ & $43,267(53.4)$ \\
\hline Rural & $151(10.2)$ & $15,641(19.3)$ \\
\hline Data missing & $138(9.3)$ & $6,923(8.5)$ \\
\hline
\end{tabular}

${ }^{a}$ Identified by using the Public Health Information Exchange (PHINEX), a database that links electronic health records collected at University of Wisconsin clinics with community-level census variables.

${ }^{\mathrm{b}}$ African-American, Asian, and other groups account for the remaining $19.3 \%$ of the PHINEX database.

${ }^{c}$ Body mass index $\left(\mathrm{kg} / \mathrm{m}^{2}\right)$ was calculated from height and weight measured on the same day. All BMI values were plotted on age-specific and sex-specific growth charts to determine BMI percentile according to the CDC 2000 charts as follows: $<5$ th percentile $=$ underweight, 5 th to $<85$ th percentile $=$ normal weight, $\geq 85$ th to $<95$ th percentile $=$ overweight, and $\geq 95$ th percentile $=$ obese (10). If multiple patient encounters were available, the most recently recorded BMI was used.

${ }^{\mathrm{d}}$ The economic hardship index is calculated at the census block group level and consists of 6 measures: crowded housing (percentage of housing units with fewer than 1 person per room), poverty (percentage of households below the federal poverty level), unemployment (percentage of people aged 16 years or older who are unemployed), education (percentage of people aged 25 years or older without a high school education), dependency (percentage of population younger than 18 years or older than 64 years), and per capita income. Scores can range from 0 to 100, with 100 indicating the highest hardship (12).

The opinions expressed by authors contributing to this journal do not necessarily reflect the opinions of the U.S. Department of Health and Human Services, the Public Health Service, the Centers for Disease Control and Prevention, or the authors' affiliated institutions. 
Table 2. Characteristics of Clinic Visits, American Indian and Non-Hispanic White Children, Taken From Electronic Health Records, University of Wisconsin Health Clinics, 2007-2012

\begin{tabular}{|l|r|r|}
\hline Clinic Visit & American Indian & Non-Hispanic White \\
\hline Visits per child per year, mean (SD) & $2.5(1.9)$ & $2.6(1.9)$ \\
\hline Diagnoses per visit, mean (SD) & $1.9(0.7)$ & $<.01$ \\
\hline Well-child visits, $\mathrm{n}(\%)$ & $767(55.9)$ & .20 \\
\hline
\end{tabular}

a Pearson $x^{2}$ or the Wilcoxon test was used to compare American Indian and non-Hispanic white children. 
Table 3. Obesity Risk in a Multivariate Model of American Indian and Non-Hispanic White Children, University of Wisconsin Health Clinics, 2007-2012

\begin{tabular}{|c|c|c|}
\hline Variable & Adjusted Odds Ratio (95\% Cl) & $P$ Value \\
\hline \multicolumn{3}{|c|}{ Economic hardship index ${ }^{a}$} \\
\hline$<20$ & $0.48(0.44-0.53)$ & $<.001$ \\
\hline $20-<25$ & $0.67(0.64-0.71)$ & $<.001$ \\
\hline$\geq 25$ & \multicolumn{2}{|r|}{1 [Reference] } \\
\hline \multicolumn{3}{|l|}{ Health insurance } \\
\hline Commercial & \multicolumn{2}{|r|}{1 [Reference] } \\
\hline Medicare or Medicaid & $1.74(1.63-1.84)$ & $<.001$ \\
\hline No insurance & $1.11(0.85-1.45)$ & .43 \\
\hline \multicolumn{3}{|l|}{ Race/ethnicity } \\
\hline American Indian & $1.85(1.60-2.14)$ & $<.001$ \\
\hline Non-Hispanic white & \multicolumn{2}{|r|}{1 [Reference] } \\
\hline \multicolumn{3}{|l|}{ Sex } \\
\hline Female & \multicolumn{2}{|r|}{1 [Reference] } \\
\hline Male & $1.31(1.25-1.38)$ & $<.001$ \\
\hline \multicolumn{3}{|l|}{ Age, y } \\
\hline $2-4$ years & $0.49(0.46-0.53)$ & $<.001$ \\
\hline $5-11$ years & $0.83(0.78-0.87)$ & $<.001$ \\
\hline $12-17$ & \multicolumn{2}{|r|}{1 [Reference] } \\
\hline \multicolumn{3}{|l|}{ Geographic designation } \\
\hline Urban & \multicolumn{2}{|r|}{1 [Reference] } \\
\hline Suburban & $0.86(0.81-0.92)$ & $<.001$ \\
\hline Rural & $0.93(0.86-0.996)$ & .04 \\
\hline
\end{tabular}

Abbreviation: $\mathrm{Cl}$, confidence interval.

${ }^{a}$ The economic hardship index is calculated at the census block group level and consists of 6 measures: crowded housing (percentage of housing units with fewer than 1 person per room), poverty (percentage of households below the federal poverty level), unemployment (percentage of people aged 16 years or older who are unemployed), education (percentage of people aged 25 years or older without a high school education), dependency (percentage of population younger than 18 years or older than 64 years), and per capita income. Scores can range from 0 to 100, with 100 indicating the highest hardship (12). 